\title{
Секвенциальные аксиоматизации пропозициональных логик нельсоновского типа ${ }^{1}$
}

\author{
В. м. Попов
}

\begin{abstract}
The sequent systems axiomatizing four Nelson's type logics are presented.

Ключевые слова: секвенция, исчисление, паранормальная логика, паранепротиворечивая логика, параполная логика
\end{abstract}

Формулируются свободные от сечения секвенциальные аксиоматизации четырех пропозициональных логик, родственных логикам, определяемым построенными в [5] и в [6] исчислениями $N$ и $N^{-}$. В [5] предложены секвенциальные исчисления $N_{s}$ и $N_{s}^{-}$, первое из которых эквиполентно исчислению $N$, а второе - исчислению $N^{-}$. Построенные ниже секвенциальные исчисления $\operatorname{GPComp}(\mathrm{N})$ и $\operatorname{GPar}(\mathrm{N})$ эквиполентны с точностью до несущественных деталей секвенциальному исчислению, являющемуся пропозициональной частью исчисления $N_{s}$, и секвенциальному исчислению, являющемуся пропозициональной частью исчисления $N_{s}^{-}$соответственно.

Язык $L$ всех рассматриваемых здесь логик есть стандартный пропозициональный язык, алфавиту которого принадлежат все следующие символы и только они: \&, $\vee, \supset$ (бинарные логические связки языка $L$ ), ᄀ (унарная логическая связка языка $L)$, ) и ( (технические символы языка $L), p_{1}, p_{2}, p_{3}, \ldots$ (пропозициональные переменные языка $L$ ). Допускаем применение обычных соглашений об опускании скобок в $L$-формулах и используем «формула» вместо «L-формула». Опишем исчисления

\footnotetext{
${ }^{1}$ Работа выполнена при поддержке РГНФ, проект № 10-03-00570а.
} 
$\operatorname{HPar}(\mathrm{N}), \operatorname{HPContPComp}(\mathrm{N}), \operatorname{HPCont}(\mathrm{N})$ и $\operatorname{HPComp}(\mathrm{N})$ гильбертовского типа, индуцирующие интересующие нас логики. Множеству всех аксиом исчисления $\operatorname{HPar}(\mathrm{N})$ принадлежат все те и только те формулы, каждая из которых имеет хотя бы один из следующих семнадцати видов (здесь и далее $A, B$ и $C$ есть формулы):

(I) $(A \supset B) \supset((B \supset C) \supset(A \supset C))$,

(II) $A \supset(A \vee B)$,

(III) $A \supset(B \vee A)$,

(IV) $(A \supset C) \supset((B \supset C) \supset((A \vee B) \supset C))$,

(V) $(A \& B) \supset A$,

(VI) $(A \& B) \supset B$

(VII) $(C \supset A) \supset((C \supset B) \supset(C \supset(A \& B)))$,

(VIII) $(A \supset(B \supset C)) \supset((A \& B) \supset C)$,

(IX) $((A \& B) \supset C) \supset(A \supset(B \supset C))$,

(X) $\neg(A \vee B) \supset(\neg A \& \neg B)$,

(XI) $(\neg A \& \neg B) \supset \neg(A \vee B)$,

(XII) $\neg(A \& B) \supset(\neg A \vee \neg B)$,

(XIII) $(\neg A \vee \neg B) \supset \neg(A \& B)$,

$(\mathrm{XIV}) \neg(A \supset B) \supset(\neg A \& B)$,

$(\mathrm{XV})(\neg A \& B) \supset \neg(A \supset B)$,

$(\mathrm{XVI}) \neg \neg A \supset A$,

(XVII) $A \supset \neg \neg A$.

Множеству всех аксиом исчисления HPContPComp(N) принадлежат все те и только те формулы, каждая из которых имеет хотя бы один из видов (I)-(XVII) или имеет вид $(A \& \neg A) \supset$ $(B \vee \neg B)$. Множеству всех аксиом исчисления $\operatorname{HPCont}(\mathrm{N})$ принадлежат все те и только формулы, каждая из которых имеет хотя бы один из видов (I)-(XVII) или имеет вид $(B \vee \neg B)$. Множеству всех аксиом исчисления $\mathrm{HPComp}(\mathrm{N})$ принадлежат все те и только формулы, каждая из которых имеет хотя бы один из видов (I)-(XVII) или имеет вид $(A \& \neg A) \supset B$. Каждое из исчислений $\operatorname{HPar}(\mathrm{N}), \operatorname{HPContPComp}(\mathrm{N}), \operatorname{HPCont}(\mathrm{N}), \operatorname{HPComp}(\mathrm{N})$ имеет единственное правило вывода - правило modus ponens в $L$. Во всяком из этих исчислений выводы (в частности, доказательства) строятся обычным для гильбертовского типа исчис- 
лений образом. Опираясь на вышеприведенные определения исчислений $\operatorname{HPar}(\mathrm{N}), \operatorname{HPContPComp}(\mathrm{N}), \operatorname{HPCont}(\mathrm{N}), \operatorname{HPComp}(\mathrm{N})$ и данные в [2] определения исчислений HPar, HPContPComp, HPCont, HPComp, замечаем, что язык всех указанных исчислений есть $L$, каждое из этих исчислений имеет единственное правило вывода - правило modus ponens в $L$, выводы (в частности, доказательства) во всех этих исчислениях строятся обычным для гильбертовского типа исчислений образом, множество всех аксиом исчисления HPar (исчисления HPContPComp, исчисления HPCont, исчисления HPComp) есть объединение множества всех аксиом исчисления $\operatorname{HPar}(\mathrm{N})$ (исчисления $\operatorname{HPContPComp}(\mathrm{N})$, исчисления $\operatorname{HPCont}(\mathrm{N})$, исчисления $\operatorname{HPComp}(\mathrm{N})$ соответственно) с множеством всех формул вида $((A \supset B) \supset A) \supset A$. Kроме того, можно доказать, что формула $\left(\left(p_{1} \supset p_{2}\right) \supset p_{1}\right) \supset p_{1}$ недоказуема ни в одном из исчислений $\operatorname{HPar}(\mathrm{N}), \operatorname{HPContPComp}(\mathrm{N})$, HPCont(N), HPComp(N). Таким образом, исчисления $\operatorname{HPar}(\mathrm{N})$, HPContPComp(N), HPCont(N), HPComp(N) являются собственными сужениями исчислений HPar, HPContPComp, HPCont, HPComp соответственно. Определяем $\operatorname{Par}(\mathrm{N})$ как множество всех формул, доказуемых в $\operatorname{HPar}(\mathrm{N})$. Аналогично определяем PContPComp $(\mathrm{N})$, PCont $(\mathrm{N})$ и $\mathrm{PComp}(\mathrm{N})$. Термины «логика», «паранепротиворечивая логика» и «паранормальная логика» далее используем в соответствии с их определениями, данными в [3]. Можно доказать, что

(a) $\operatorname{Par}(\mathrm{N})$ и $\mathrm{PCont} \operatorname{PComp}(\mathrm{N})$ есть различные паранормальные логики, при этом $\operatorname{Par}(\mathrm{N}) \subseteq \operatorname{PContPComp}(\mathrm{N})$,

(б) $\mathrm{PCont}(\mathrm{N})$ есть паранепротиворечивая, но не параполная логика,

(в) $\mathrm{PComp}(\mathrm{N})$ есть параполная, но не паранепротиворечивая логика,

(г) $\operatorname{PContPComp}(\mathrm{N}) \subseteq \operatorname{PCont}(\mathrm{N}) \cap \operatorname{PComp}(\mathrm{N})$, но PContPComp $(\mathrm{N})$ и $\mathrm{PCont}(\mathrm{N}) \cap \mathrm{PComp}(\mathrm{N})$ не равны.

Приступим теперь к конструированию секвенциальных исчислений $\operatorname{GPar}(\mathrm{N}), \operatorname{GPContPComp}(\mathrm{N}), \operatorname{GPCont}(\mathrm{N}), \operatorname{GPComp}(\mathrm{N})$. Построение каждого из этих исчислений аналогично построению исчисления GPContPComp в работе [3]. Обозначая через 
ВИП(N) секвенциальное правило, являющееся множеством всех упорядоченных пар вида $<(A \bullet \Gamma) \rightarrow B, \Gamma \rightarrow A \supset B>$, где $\Gamma-$ последовательность формул, определяем множество всех правил вывода каждого из исчислений $\operatorname{GPar}(\mathrm{N}), \operatorname{GPContPComp}(\mathrm{N})$, $\operatorname{GPCont}(\mathrm{N})$ и $\operatorname{GPComp}(\mathrm{N})$ как такое множество $\mathrm{M}$, что $\Pi \in \mathrm{M}$ тогда и только тогда, когда П есть ВИП(N) или любое, исключая ВИП, правило вывода исчисления GPContPComp. Множество всех основных секвенций исчисления $\operatorname{GPar}(\mathrm{N})$ есть множество всех секвенций вида $A \rightarrow A$. Множество всех основных секвенций исчисления GPContPComp (N) есть объединение множества всех основных секвенций исчисления $\operatorname{GPar}(\mathrm{N})$ с множеством всех секвенций вида $A, \neg A \rightarrow B, \neg B$. Множество всех основных секвенций исчисления GPCont $(\mathrm{N})$ есть объединение множества всех основных секвенций исчисления $\operatorname{GPar}(\mathrm{N})$ с множеством всех секвенций вида $\rightarrow A, \neg A$. Множество всех основных секвенций исчисления $\operatorname{GPComp}(\mathrm{N})$ есть объединение множества всех основных секвенций исчисления $\operatorname{GPar}(\mathrm{N})$ с множеством всех секвенций вида $A, \neg A \rightarrow$. Выводы во всех секвенциальных исчислениях $\operatorname{GPar}(\mathrm{N}), \operatorname{GPContPComp}(\mathrm{N}), \operatorname{GPCont}(\mathrm{N})$ и GPComp(N) строятся обычным для этого типа исчислений образом. Для каждого из этих исчислений доказана теорема об устранимости сечения. С использованием этой теоремы показано, что исчисления $\operatorname{GPar}(\mathrm{N}), \operatorname{GPContPComp}(\mathrm{N}), \operatorname{GPCont}(\mathrm{N})$, $\operatorname{GPComp}(\mathrm{N})$ являются секвенциальными аксиоматизациями логик $\operatorname{Par}(\mathrm{N}), \operatorname{PContPComp}(\mathrm{N}), \operatorname{PCont}(\mathrm{N})$, PComp(N), соответственно. Точнее, доказана теорема о том, что для всякой логики $\mathbf{L}$ из $\{\operatorname{Par}(\mathrm{N}), \operatorname{PContPComp}(\mathrm{N}), \operatorname{PCont}(\mathrm{N}), \operatorname{PComp}(\mathrm{N})\}$ и для всякой формулы $A$ верно, что секвенция $\rightarrow A$ выводима в GL тогда и только тогда, когда $A \in \mathbf{L}$. Можно доказать также следующее утверждение: позитивный фрагмент любой из логик $\operatorname{Par}(\mathrm{N}), \operatorname{PContPComp}(\mathrm{N}), \operatorname{PCont}(\mathrm{N}), \operatorname{PComp}(\mathrm{N})$ является позитивным фрагментом интуиционистской пропозициональной логики, язык которой есть $L$. В свете утверждения ясно, что ни одна из логик $\operatorname{Par}(\mathrm{N}), \operatorname{PContPComp}(\mathrm{N}), \operatorname{PCont}(\mathrm{N}), \operatorname{PComp}(\mathrm{N})$ не имеет конечной характеристической матрицы. Тем не менее, все эти логики разрешимы. Разрешимость любой из указанных логик вытекает из разрешимости построенного выше секвенциального исчисления, аксиоматизирующего эту логику. В свою 
очередь, для каждого исчисления $\operatorname{GPar}(\mathrm{N}), \operatorname{GPContPComp}(\mathrm{N})$, GPCont(N), GPComp(N) разрешимость доказывается генценовским методом, который впервые применен в [1]. При этом в ходе применения генценовского метода для доказательства разрешимости исчисления $\mathbf{G}$ из $\{\operatorname{GPar}(\mathrm{N}), \operatorname{GPContPComp}(\mathrm{N}), \operatorname{GPCont}(\mathrm{N})$, $\operatorname{GPComp}(\mathrm{N})\}$ используется нижеследующая теорема об обобщенной подформульности $\mathbf{G - в ы в о д о в , ~ а ~ н е ~ а н а л о г ~ т е о р е м ы ~} 2.513$ из [1] (теоремы о подформульности $L I$-выводов и $L K$-выводов).

TEOPEMA. Пусть $\mathbf{G} \in\{\operatorname{GPar}(\mathrm{N})$, GPContPComp $(\mathrm{N})$, GPCont $(\mathrm{N})$, $\operatorname{GPComp}(\mathrm{N})\}$.

Если формула А есть подформула формуль, входлщей в неко-

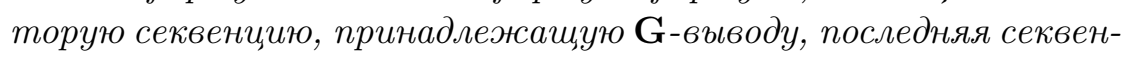
иия которого есть $S$, то

(1) если $A$ не имеет вид $\neg B$ ни для какой формуль $B$, то $A$ есть подформула некоторой формуль, входящей в $S$,

(2) если $A$ имеет вид $\neg B$ для некоторой формуль $B$, то по крайней мере одна из формул $B$ или $\neg B$ есть подформула некоторой формуль, входящей в $S$.

В заключение заметим, что естественно возникающая проблема построения свободной от сечения секвенциальной аксиоматизации логики $\mathrm{PCont}(\mathrm{N}) \cap \mathrm{PComp}(\mathrm{N})$ открыта.

\section{Литература}

[1] Генщен Г. Исследования логических выводов // Математическая теория логического вывода. М., 1967. С.9-74.

[2] Попов B.M. Между Раг и множеством всех формул // Шестые смирновские чтения по логике. Материалы международной научной конференции 17-19 июня 2009. М., 2009. С. 93-95.

[3] Попов B.M. Секвенциальная аксиоматизация паранормальной логики PContPComp // В настоящем сборнике.

[4] Смирнов B.A. Формальный вывод и логические исчисления//Смирнов В.А. Теория логического вывода. М., 1999. С. 16-233.

[5] Almukdad A., Nelson D. Constructible falsity and inexact predicates // J. Symb. Log. 1984. Vol. 49. № 1. P. 231-233.

[6] Nelson D. Constructible falsity // J. Symb. Log. 1949. Vol. 14. № 1. P. 16-26. 